\title{
Impact of incorporated oxygen quantity on optical, structural and dielectric properties of reactive magnetron sputter grown high- $\kappa$ $\mathrm{HfO}_{2} / \mathrm{Hf} / \mathrm{Si}$ thin film
}

\author{
A. Cantas ${ }^{\mathrm{a}}, \mathrm{G}$. Aygun $^{\mathrm{a}, *}$, R. Turan ${ }^{\mathrm{b}}$ \\ a Department of Physics, Izmir Institute of Technology, TR-35430 Urla, Izmir, Turkey \\ ${ }^{\mathrm{b}}$ Department of Physics, Middle East Technical University, TR-06531 Ankara, Turkey
}

\section{A R T I C L E I N F O}

\section{Article history:}

Received 31 October 2013

Received in revised form 12 March 2014

Accepted 12 March 2014

Available online 20 March 2014

\section{Keywords:}

$\mathrm{HfO}_{2}$

High-к dielectric

Reactive rf sputtering

In situ SE

FTIR

GIXRD

\begin{abstract}
A B S T R A C T
High- $\kappa$ hafnium-oxide thin films have been fabricated by radio frequency (rf) reactive magnetron sputtering technique. To avoid formation of an undesired interfacial suboxide layer between Si and high- $\kappa$ film, prior to $\mathrm{HfO}_{2}$ deposition, a thin $\mathrm{Hf}$ buffer layer was deposited on $p$-type (100) Si substrate at room temperature. Effect of oxygen gas quantity in the $\mathrm{O}_{2} / \mathrm{Ar}$ gas mixture was studied for the optical and structural properties of grown $\mathrm{HfO}_{2}$ high- $\kappa$ thin films. The grown thin oxide films were characterized optically using spectroscopic ellipsometer (SE) in detail. Crystal structure was studied by grazing incidence X-ray diffractometer (GIXRD) technique, while bonding structure was obtained by Fourier transform infrared spectroscopy (FTIR) analyses. In agreement with GIXRD and FTIR analyses, SE results show that any increment above ideal quantity of oxygen content in the gas mixture resulted in decrements in the refractive index and thickness of $\mathrm{HfO}_{2}$ dielectric film, while increments in $\mathrm{SiO}_{2}$ thickness. It is apparent from experimental results that oxygen to argon gas ratio needs to be smaller than 0.2 for a good film quality. The superior structural and optical properties for grown oxide film were obtained for $\mathrm{O}_{2} / \mathrm{Ar}$ gas ratio of about 0.05-0.1 combined with $\sim 30 \mathrm{~W}$ constant rf sputtering power.
\end{abstract}

(C) 2014 Elsevier B.V. All rights reserved.

\section{Introduction}

It is commonly known that $\mathrm{SiO}_{2}$, silicon oxynitride $\left(\mathrm{SiO}_{x} \mathrm{~N}_{y}\right)$, and $\mathrm{Si}_{3} \mathrm{~N}_{4}$ gate dielectric materials for ultra-large scale integration (ULSI) need to be replaced by high- $\kappa$ dielectric films due to their leakage current problem below $2 \mathrm{~nm}$ thickness [1,2]. Recently, $\mathrm{HfO}_{2}$ has been proposed as a replacement since it has a large bandgap $\left(E_{\mathrm{g}}>5 \mathrm{eV}\right)$ as well as high dielectric value of $\sim 25$ and thermodynamically stable when grown on Si [3,4]. Moreover, having high refractive index value and good transmission characteristics in the visible spectrum range, $\mathrm{HfO}_{2}$ has been of interest for antireflective multilayer coating technology [5,6]. Another important aspect of this novel material is its application on thermal barrier coatings for turbine blades operating in harsh and high temperature ambient due to its high melting temperature $\left(\sim 2800^{\circ} \mathrm{C}\right)$ and thermal stability [7].

A number of compatible methods have been developed to fabricate high- $\kappa$ materials such as thermal oxidation [8], a variety

\footnotetext{
* Corresponding author. Tel.: +90 232750 7715; fax: +90 2327507707 .

E-mail address: gulnuraygun@iyte.edu.tr (G. Aygun).
}

of chemical vapor deposition techniques [9], ion beam deposition [10], atomic layer deposition [11,12], pulsed laser deposition [13], laser oxidation [14,15], remote plasma oxidation [16], dc and rf sputtering $[8,17,18]$. Among them, magnetron sputtering technique has attracted special attention due to its high growth rate, good control over physical properties of grown film and ability to grow in amorphous phase.

However, a common problem in all production methods is that an unavoidable interfacial layer formation occurs between the grown high-к film and Si substrate [19]. Since many film properties like dielectric constant and refractive index depend on the thin film composition, various research groups have been trying different methods in order to prevent formation of this undesired interfacial layer [17]. It was shown in the literature that the physical parameters during oxidation are crucially important on the quality of grown film [20,21]. Recently, we examined thin Hf-oxide films grown on Si substrate by d.c. reactive sputtering method using XPS depth profiling experiments [22]. We showed that, being highly reactive with oxygen radicals, the sputtered Hf atoms consume some of the oxygen constitutions from the underlying native $\mathrm{SiO}_{2}$ film in order to form Hafnium-oxide and leave either elemental Si or $\mathrm{Si}-\mathrm{Si}$ bonds behind. 
The aim of this study is not only to understand the oxygen impact on the grown high- $\kappa$ thin films' quality, but also to determine the dependence of the incorporated oxygen quantity on the formation of an undesired interfacial layer having a lower refractive index between the Si substrate and high- $\kappa$ thin oxide film. Therefore, suboxide formation has been tried to be eliminated by depositing a thin $\mathrm{Hf}$ buffer prior to reactive oxidation of $\mathrm{Hf}$ in $\mathrm{Ar}$ and $\mathrm{O}_{2}$ environment to grow $\mathrm{HfO}_{2}$ thin film on $\mathrm{Si}$ substrate. We aimed that thin Hf buffer would interact with underlying native oxide of $\mathrm{Si}$ to diminish its oxygen atoms just leaving Si substrate behind [22]. In summary, we grew initially a thin Hf buffer layer on Si substrate, later focused on the importance of oxygen quantity variation in the $\mathrm{O}_{2} / \mathrm{Ar}$ gas ratio during radio frequency $(\mathrm{RF})$ reactive sputtering of thin $\mathrm{HfO}_{2}$ films.

\section{Experimental details}

$p$-Type (1 000$)$ Si wafers with a resistivity of $7-17 \Omega$-cm were used as substrates. Initially, they were etched for $30 \mathrm{~s}$ in a $1 \%$ hydrofluoric (HF) acid in order to remove the silicon's native oxide from Si substrates. Then, they were rinsed in de-ionized water, dried with pure $\mathrm{N}_{2}$ gas and mounted onto the substrate holder having a $7.6 \mathrm{~cm}$ distance to the target of magneto-sputtering deposition chamber vacuumed below $10^{-6}$ Torr with a turbo molecular pump (TMP). A 2 "diameter with 0.25 " thick of $99.9 \%$ pure hafnium was used as the sputtering target. In spite of its high purity, an additional pre-sputtering with Ar gas flow for $1 \mathrm{~min}$ was realized to remove the possible surface contaminants from the target surface. Ar gas flow was held constant at $30 \mathrm{sccm}$ for all experiments. During sputtering, not any intentional substrate heating was applied and the working gas pressure was $0.44 \times 10^{-3}$ Torr.

Thin film deposition process consists of two growth stages. Initially, a thin Hf buffer layer was deposited on chemically cleaned Si substrate in order to be able to avoid the formation of any undesired interfacial suboxide layer $[8,17,22]$. Thickness of Hf buffer layer grown for $60 \mathrm{~s}$ with $30 \mathrm{~W}$ sputtering power was measured $5 \mathrm{~nm}$ by a profilometer. Following Hf buffer layer deposition on Si substrate, oxygen as being the reactive gas was sent into the growth chamber in a controlled manner, additional to Ar sputtering gas. The first group of samples were grown with $30 \mathrm{~W}$ constant rf power with variable oxygen quantity between 0.75 and $15 \mathrm{sccm}$ to obtain $\mathrm{O}_{2}$ to Ar gas ratio from 0.025 to 0.5 . The second group of films were grown with $7.5 \mathrm{sccm}$ constant amount of oxygen with different rf power ranging from $30 \mathrm{~W}$ to $60 \mathrm{~W}$. Sputtering time was kept at $3 \mathrm{~min}$ for the growth processes. The flow chart for the experimental process is given in Fig. 1.

Fig. 2 shows the schematic view of experimental setup. Spectroscopic ellipsometer (SE) mounted onto the sputtering chamber was used to evaluate the optical parameters, i.e. refractive index and dielectric constant as well as thickness of grown hafnium oxide film. SE measurements were made at $70^{\circ}$ fixed incident angle in the wavelength range of $300-850 \mathrm{~nm}$ by SENTECH SE-801. SpectraRay program was used for modeling and fitting processes of SE data to determine the refractive index $(n)$, thickness $(d)$ and real part of dielectric constant $\left(\varepsilon_{1}\right)$. Thickness and refractive index of $\mathrm{HfO}_{2}$ and $\mathrm{SiO}_{2}$, and dielectric constant of $\mathrm{HfO}_{2}$ obtained from SE measurements were compared according to their $\mathrm{O}_{2} / \mathrm{Ar}$ gas ratio for the first group and different rf power for the second group.

Crystalline structure of grown films were examined by taking grazing incidence X-ray diffraction (GIXRD) of a thin film XRD system (PANALITICAL X'Pert Pro MRD) using $C u K_{\alpha}$ line with the wavelength of $1.5402 \AA$. The XRD patterns were analyzed by means of a computer software program including ICDD database with the diffraction patterns of well known structures. The peak matching

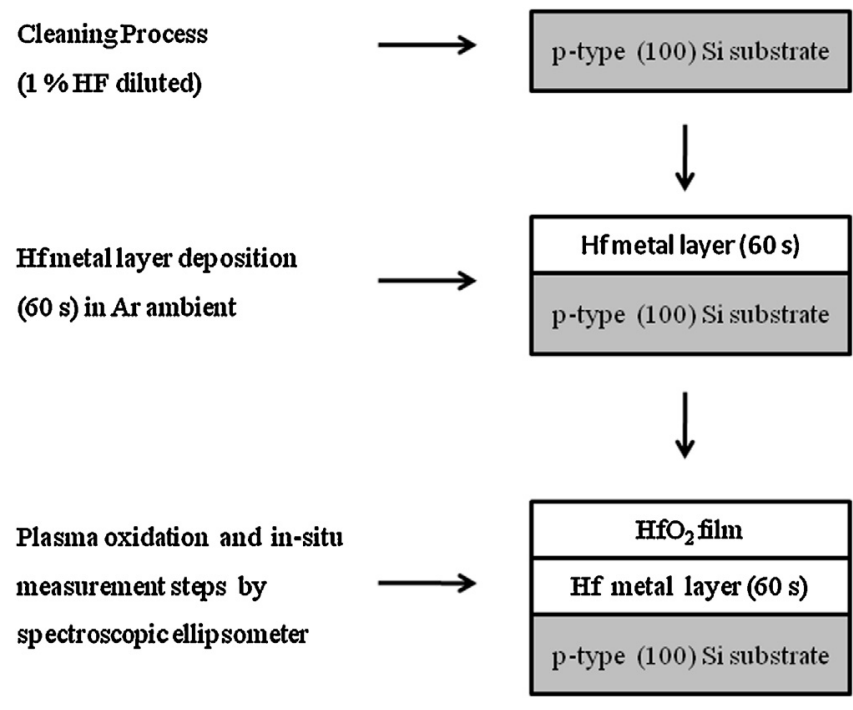

Fig. 1. The details of experimental process flow chart.

process was carried out with relative intensities of the peaks based on the observed peak positions at specific $2 \theta$ values.

A BRUKER Equinox 55 type Fourier transform infrared (FTIR) spectrometer was used to obtain information on both the chemical composition and the structure of the films. The bonding structure of the films was studied in the range of $1200-400 \mathrm{~cm}^{-1}$ by FTIR spectroscopy.

\section{Results and discussion}

\subsection{XRD structural properties}

Grazing incidence X-ray diffraction (GIXRD) measurements of $\mathrm{HfO}_{2}$ thin films were measured with $0.5^{\circ}$ grazing angle from $10^{\circ}$ to $65^{\circ}$ in the $2 \theta$ range at room temperature (Fig. 3(a)). XRD peaks were matched to the ones from ICDD (International Center for Diffraction Data) [23]. It is barely visible that the films have an extremely broad signal corresponding to amorphous $\mathrm{HfO}_{2}\left(2 \theta \sim 32^{\circ}\right)$ [23-25]. The $2 \theta$ peaks at $\sim 51.6^{\circ}$ and $53.8^{\circ}$ are attributed, respectively, to (-2 21$)$ monoclinic and (023) orthorhombic phases of $\mathrm{HfO}_{2}$.

Formation of polycrystalline structure can be inferred from XRD plot having a very low peak intensity combined with a high FWHM. Since the spectrum has a very broad peak ranging between $54.1^{\circ}$ and $57.5^{\circ}$ with low intensity, it corresponds to the combination of a few polycrystalline structures of $\mathrm{HfO}_{2}$ [23]. Since the crystalline state affects leakage current levels of materials in a negative manner [14], it is not a desired material property for high-к dielectrics [26]. It is obvious that the film grown with the oxygen to argon gas ratio content of 0.1 shows very weak crystallization state while the peak intensities increase as the referred gas ratio content increases from 0.1 to 0.5 .

Fig. 3(b) shows the crystalline structure of sputter grown thin hafnium-oxide films depending on applied sputtering power. Since $\mathrm{HfO}_{2}$ is used as an insulator in microelectronic technology, obtaining its amorphous structure is important. It is clearly seen that as the sputtering power is increased, the crystallization of Hf-oxide film also increases. It is inferred for sputtering power of $30 \mathrm{~W}$ that not only the peak at $51.6^{\circ}$ is absent, but also the peak about $53.8^{\circ}$ has the lowest intensity. This implies that the lowest level of crystalline state is detected for $30 \mathrm{~W}$ of sputtering power. Also, we observed that the films grown at higher sputtering powers than that of $30 \mathrm{~W}$ have a very broad and low intensity signal of (111) monoclinic phase of $\mathrm{HfO}_{2}\left(2 \theta \sim 32^{\circ}\right)[19,24,25]$. Since the sputtered Hf 


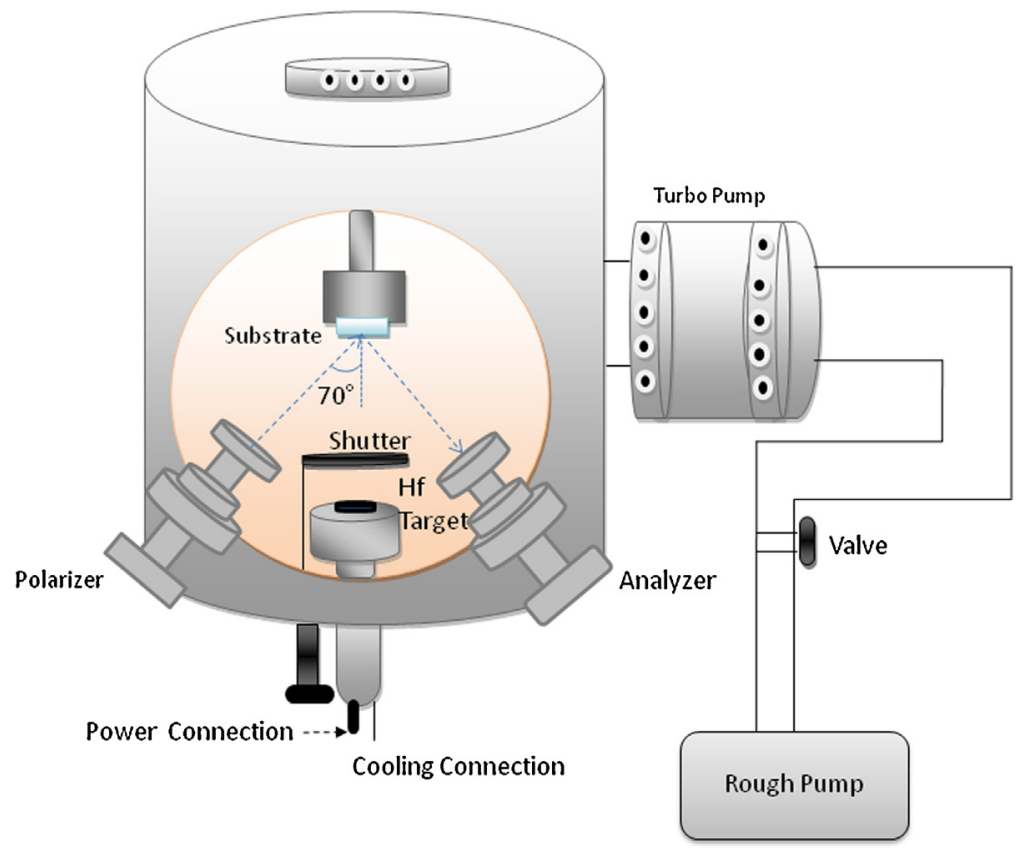

Fig. 2. Schematic view of sputtering growth chamber combined with an in situ SE system.
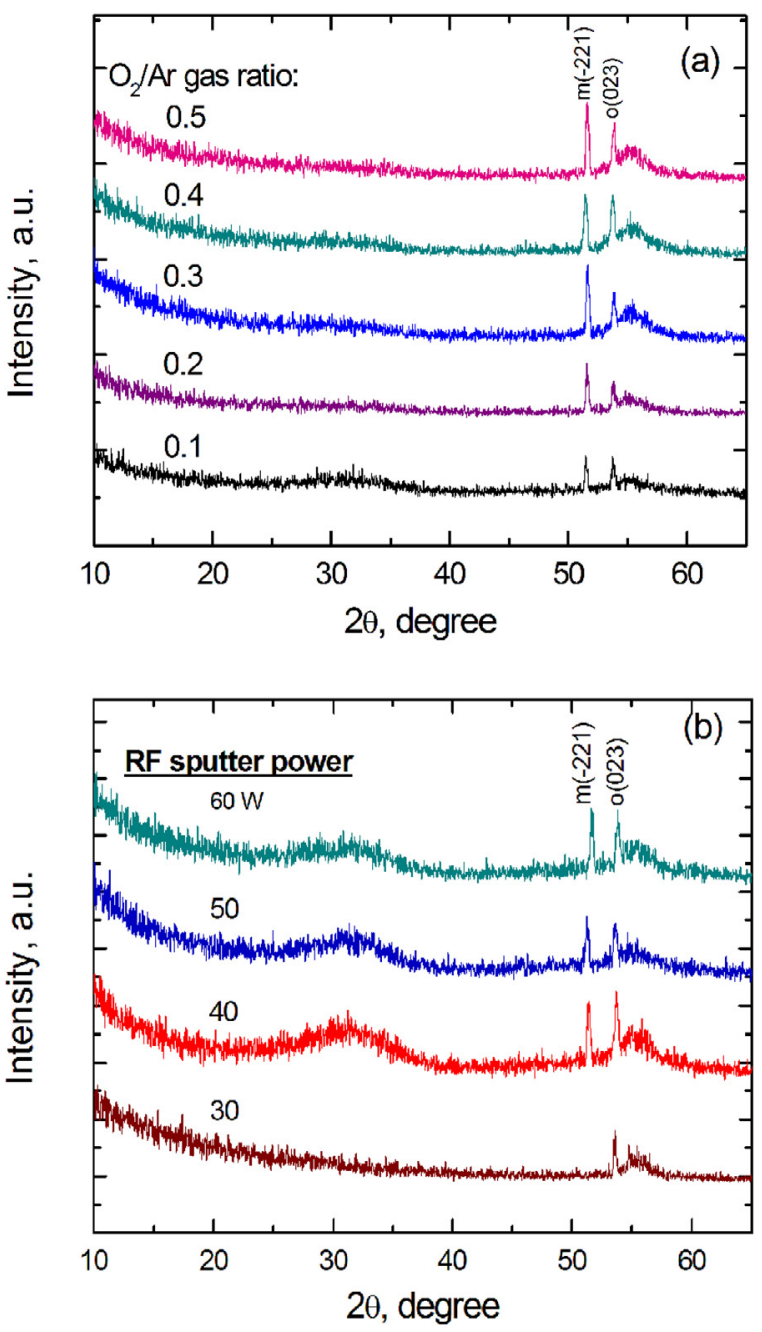

Fig. 3. GIXRD patterns for $\mathrm{HfO}_{2}$ films deposited at $\mathrm{O}_{2} / \mathrm{Ar}=0.25$ for (a) different amount of $\mathrm{O}_{2} / \mathrm{Ar}$ gas ratio with $\mathrm{P}=30 \mathrm{~W}$ and (b) different sputtering powers. particles under higher sputtering powers gain increased velocities, they probably transfer heat onto the film surface resulting in a possible temperature increment of film surface. It is well known from literature that the substrate heating generally results in crystallization of grown film $[20,26,27]$, which is undesirable for high- $\kappa$ dielectrics because of the formation of leakage current in the films $[14,28,29]$. Even though the substrate is not intentionally heated during the deposition and the sputtering is realized at room temperature, an unintentional heating of film's surface take place as a result of increased sputtering power giving rise to a crystalline thin film structure. The second possible consequence of the elevated sputter power on the grown film is the increase of porosity. Void concentration increments as a result of higher rf sputtering power levels was also reported by Pereira et al. [29]. As a result of high substrate temperature, the diffusion probability for the reactive oxygen atoms increases giving rise to interaction with Si substrate and formation of the oxide layer at the interface. Such an interfacial layer formation was previously verified by SE and FTIR analyses [22].

\subsection{FTIR spectra of $r f$ sputtered $\mathrm{HfO}_{2}$ films}

Fig. 4(a) shows Fourier transform infrared (FTIR) spectrum of Hf oxide film grown under different oxygen to argon gas ratios ranging from 0.1 to 0.5 . The bonding structures of $\mathrm{HfO}_{2}$ films are detected in the $7500-370 \mathrm{~cm}^{-1}$, but the important features are seen in the $400-1200 \mathrm{~cm}^{-1}$ spectral region. To observe only the effect of grown film, the infrared absorption spectra of substrate were taken as background signal and subtracted from the measurement.

FTIR spectrum for 0.1 gas ratio choice has no features in the expected region while FTIR spectrum of 0.2 gas ratio has a hardly visible broadband peak which is centered about $1080 \mathrm{~cm}^{-1}$. It corresponds to transverse optical component of the asymmetric stretch of $\mathrm{SiO}_{4}[30,31]$. Neumayer et al. observed a broad absorption band between 1200 and $810 \mathrm{~cm}^{-1}$ [30]. They stated that it was composed from three components namely, asymmetric stretch of $\mathrm{SiO}_{4}$ between 1180 and $1080 \mathrm{~cm}^{-1}$ [32], a shoulder at $\sim 970 \mathrm{~cm}^{-1}$ assigned to $\mathrm{HfSiO}$ and a shoulder at $\sim 880 \mathrm{~cm}^{-1}$ assigned to $\mathrm{Si}-\mathrm{O}^{-}\left(\mathrm{O}^{-}\right.$is the non bridging oxygen ion bonded to a proton $)$. FTIR spectra of the films grown with the gas ratio higher than 0.2 

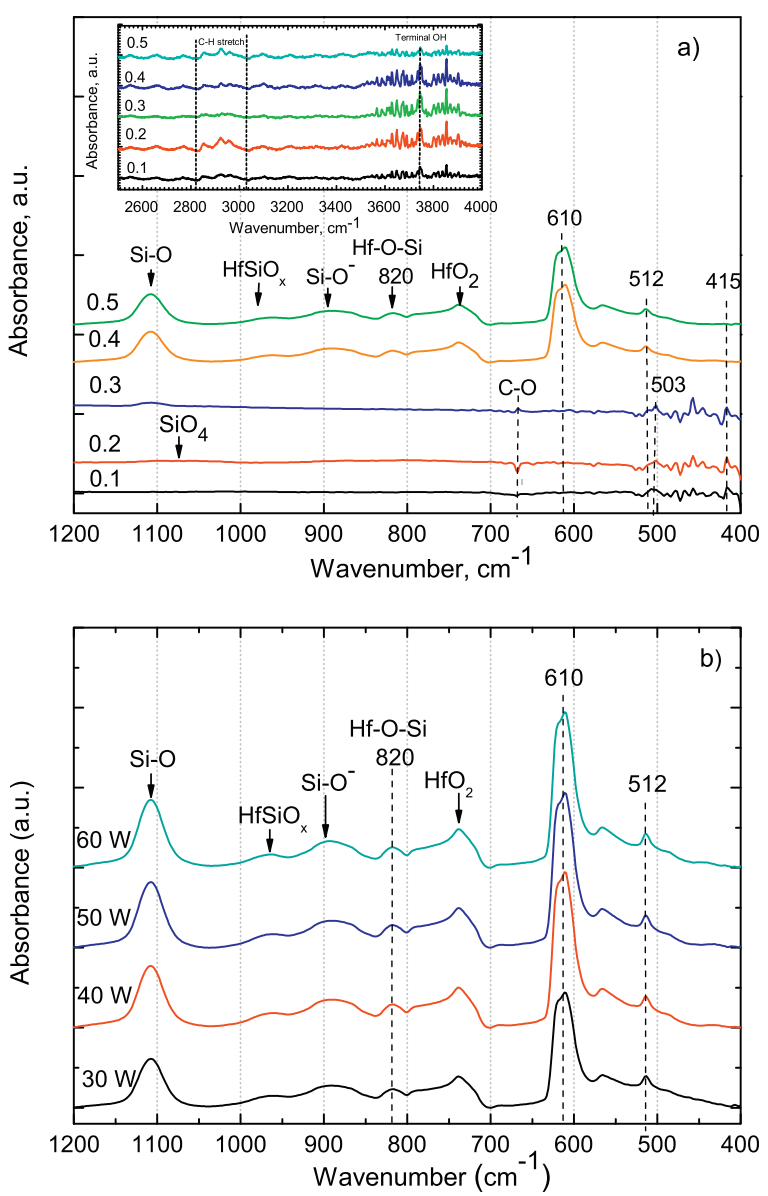

Fig. 4. FTIR spectrum of sputter grown thin high-к $\mathrm{HfO}_{2}$ layers with respect to (a) $\mathrm{O}_{2} / \mathrm{Ar}$ gas ratio levels at $\mathrm{P}=30 \mathrm{~W}$, (b) applied power levels at $\mathrm{O}_{2} / \mathrm{Ar}$ gas ratio 0.25 .

show some specific features. A peak located around $1108 \mathrm{~cm}^{-1}$ is observed with increasing intensity with respect to the increments in the gas ratio of $0.3,0.4$ and 0.5 corresponds to $\mathrm{Si}-\mathrm{O}$ vibration. According to the report of Alers et al., the absorption peak located around $1105 \mathrm{~cm}^{-1}$ assigned to interstitial oxygen in the $\mathrm{Si}$ bulk [33]. Even though no features corresponding to $\mathrm{HfSiO}_{x}$ were detected in $960-980 \mathrm{~cm}^{-1}$ for the film grown with 0.3 gas ratio, some peaks were observed for the films grown with 0.4 and 0.5 gas ratios [11,34]. The peak at $820 \mathrm{~cm}^{-1}$ is commonly attributed to $\mathrm{Hf}-\mathrm{O}-\mathrm{Si}$ stretching vibrations and the one lying from $934 \mathrm{~cm}^{-1}$ to $838 \mathrm{~cm}^{-1}$ corresponds for $\mathrm{Si}-\mathrm{O}^{-}$[35]. It is clear that when the oxygen content increase in $\mathrm{HfO}_{2}$, intensity of the absorption peak attributed to $\mathrm{HfSiO}_{x}$ becomes strong as well as the $\mathrm{Si}-\mathrm{O}$ vibration. As a result of an increase in the amount of incorporated oxygen, more oxygen may be adsorbed on the film surface during deposition, later these oxygen radicals penetrate from the deposited layer to the $\mathrm{Si}$ substrate to form the $\mathrm{SiO}_{x}$ and $\mathrm{HfSiO}_{x}$ interface. The strong peak observed at $610 \mathrm{~cm}^{-1}$ for the film grown with 0.4 and 0.5 gas ratio is related to absorption of a Si phonon [31] and also the broad peak lying from 758 to $703 \mathrm{~cm}^{-1}$ consists of two convoluted peaks located about $720 \mathrm{~cm}^{-1}$ and $748 \mathrm{~cm}^{-1}$ corresponding to $\mathrm{HfO}_{2}$ [31]. The other main peaks around 512,503 and $415 \mathrm{~cm}^{-1}$ are due to Hf-O chemical bonds [22,30,36].

To determine $\mathrm{C}-\mathrm{H}$ bonding and water molecules adsorbed in the film, FTIR spectrum from 2500 to $4000 \mathrm{~cm}^{-1}$ under vacuum conditions was realized for the films grown with different $\mathrm{O}_{2} / \mathrm{Ar}$ gas ratio. The broad and weak peak detected between 2820 and $3027 \mathrm{~cm}^{-1}$ is assigned to the $\mathrm{C}-\mathrm{H}$ bonding $[30,36]$. The peak positioned at $3743 \mathrm{~cm}^{-1}$ is attributed to terminal $\mathrm{O}-\mathrm{H}$ peak.
Fig. 4(b) shows FTIR spectrum of $\mathrm{HfO}_{2}$ samples with respect to applied sputtering power at 0.25 gas ratio. It is remarkable that the intensity of peak at $610 \mathrm{~cm}^{-1}$ representing absorption of a $\mathrm{Si}$ phonon increases with applied power. Additionally, the single peak at $512 \mathrm{~cm}^{-1}$ for $\mathrm{Hf}-\mathrm{O}$ and the peak at $1108 \mathrm{~cm}^{-1}$ corresponding to $\mathrm{Si}-\mathrm{O}$ vibration were observed for all samples. As a result, it can be inferred that different applied power has a noticeable effect on Si phonon vibration.

\subsection{Spectroscopic ellipsometer (SE)}

Complex refractive index, $N=n+i k$, and dielectric constant, $\varepsilon=\varepsilon_{1}+i \varepsilon_{2}$, describe the optical properties of any medium. The real and imaginary components of complex refractive index, respectively, $n$ and $k$ are called the refractive index and extinction coefficient. Relations between real and imaginary parts of complex refractive index and dielectric constant are given by:

$$
\begin{aligned}
& \varepsilon_{1}=n^{2}-k^{2} \text { and } \varepsilon_{2}=2 n k \\
& k=\frac{\lambda}{4 \pi} \alpha \\
& I(x)=I_{0} e^{-a x}
\end{aligned}
$$

where $\alpha$ is absorption coefficient and $I$ is intensity of light in the dielectric material from which light is passing through. The five parameters, $n, k, \varepsilon_{1}, \varepsilon_{2}$ and $\alpha$ define the optical characteristics and they are, in the mean time, related to the electronic properties of the medium.

Measurement technique of an ellipsometer is based on the changes in polarization state during reflection off of a light from a film surface at an oblique incidence angle, $\phi$ [37]. Complex reflection ratio, $\rho$, is obtained as an outcome of matching the electric and magnetic fields at the interface between different materials;

$\rho=\frac{r_{p}}{r_{s}}=\frac{\left|r_{p}\right|}{\left|r_{s}\right|} e^{i\left(\delta_{p}-\delta_{s}\right)}=\tan \Psi e^{i \Delta}$

where $\psi, \Delta$ and $\rho$ are the parameters of an ellipsometer measurement. The relation between optical and electrical parameters of bulk materials is found as:

$\varepsilon=\varepsilon_{1}+i \varepsilon_{2}=\sin ^{2} \phi\left[1+\tan ^{2} \phi\left(\frac{1-\rho}{1+\rho}\right)^{2}\right]$

Dielectric constant of any material is given by Eq. (5). Real part, $\varepsilon_{1}$, and imaginary part, $\varepsilon_{2}$, of dielectric constant define, respectively, the transparency and absorbance of the interested material. Since $\mathrm{HfO}_{2}$ is transparent to the used wavelength range of SE measurements, imaginary part, $\varepsilon_{2}$ vanishes.

$\mathrm{SE}$ measurements of $\mathrm{HfO}_{2}$ thin film grown on $p$-type (1 000$) \mathrm{Si}$ were realized with respect to wavelength over the spectral range of $300-850 \mathrm{~nm}$ at $70^{\circ}$ fixed incidence angle of light [22]. Ellipsometrically measured parameters, i.e., $\psi$ and $\Delta$, are fitted to the calculated ones using a suitable modeling in order to determine the film thickness and optical constants of the layer.

Since the sputtered grown $\mathrm{HfO}_{2}$ thin film and $\mathrm{SiO}_{2}$ interfacial layer are optically transparent to the light used in the spectral range of interest, i.e. $\lambda>350 \mathrm{~nm}$, Cauchy dispersion relation was used for the modeling step. It is well known that the Cauchy dispersive model is usually being used to fit ellipsometric data for dielectrics and semiconductors in spectral regions where they are transparent (i.e. where the extinction coefficient is zero) $[36,38]$. This model allows determination of optical coefficients (i.e. refractive index, dielectric constant) and thickness of analyzed layers of given structure. After having constructed the model (Fig. 5), the parameters, namely, thickness and refractive index of $\mathrm{HfO}_{2}$ thin high-к layer as well as thickness of $\mathrm{SiO}_{2}$ interfacial layer were left as fitting variables. Measurements were taken from $300 \mathrm{~nm}$ to $850 \mathrm{~nm}$ 

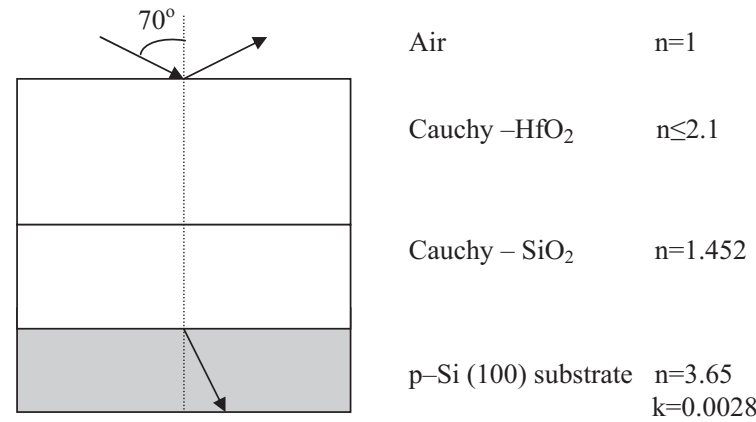

Fig. 5. Modeling used for SE of the grown film layers on $p$-Si (1 00$)$ substrate.

Table 1

MSE values for thickness of $\mathrm{HfO}_{2}$ film grown at $30 \mathrm{~W}$.

\begin{tabular}{ll}
\hline $\mathrm{O}_{2} / \mathrm{Ar}$ & Mean squared error for thickness of $\mathrm{HfO}_{2}$ \\
\hline 0.1 & 0.64 \\
0.2 & 0.50 \\
0.3 & 0.49 \\
0.4 & 0.38 \\
0.5 & 0.47 \\
\hline
\end{tabular}

Table 2

MSE values for thickness of $\mathrm{HfO}_{2}$ film grown at $0.25 \mathrm{O}_{2} / \mathrm{Ar}$ gas ratio.

\begin{tabular}{ll}
\hline Power & Mean squared error for thickness of $\mathrm{HfO}_{2}$ \\
\hline 30 & 1.08 \\
40 & 1.05 \\
50 & 0.88 \\
60 & 1.75 \\
\hline
\end{tabular}

wavelength range, but fitting was performed at the wavelength of $632 \mathrm{~nm}$. Table 1 shows the mean-squared error (MSE) error for $\mathrm{HfO}_{2}$ thickness with respect to variable gas ratios while Table 2 shows MSE errors with respect to sputter power values for the thickness of $\mathrm{HfO}_{2}$ films.

We begin with the optimization of sputtering power for $\mathrm{HfO}_{2}$. The effect of applied sputter power increments on the refractive index of $\mathrm{HfO}_{2}$ layers is shown in Fig. 6 and on the crystalline structure is given in Fig. 3(b). It is seen from Fig. 6 that when the rf sputtering power is increased gradually from $30 \mathrm{~W}$ to $60 \mathrm{~W}$, the refractive index of grown Hf-oxide layer decreases. This result may probably be explained in a few possible ways. Since the

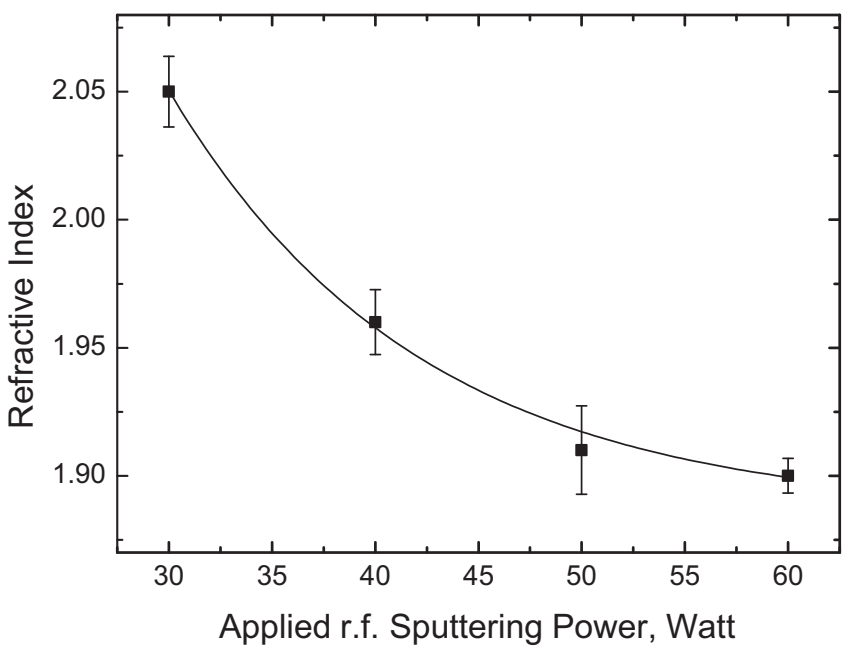

Fig. 6. Dependence of refractive index, $n$, at the wavelength of $632 \mathrm{~nm}$ on applied sputtering power. Gas ratio of 0.25 was used for all cases of this plot.
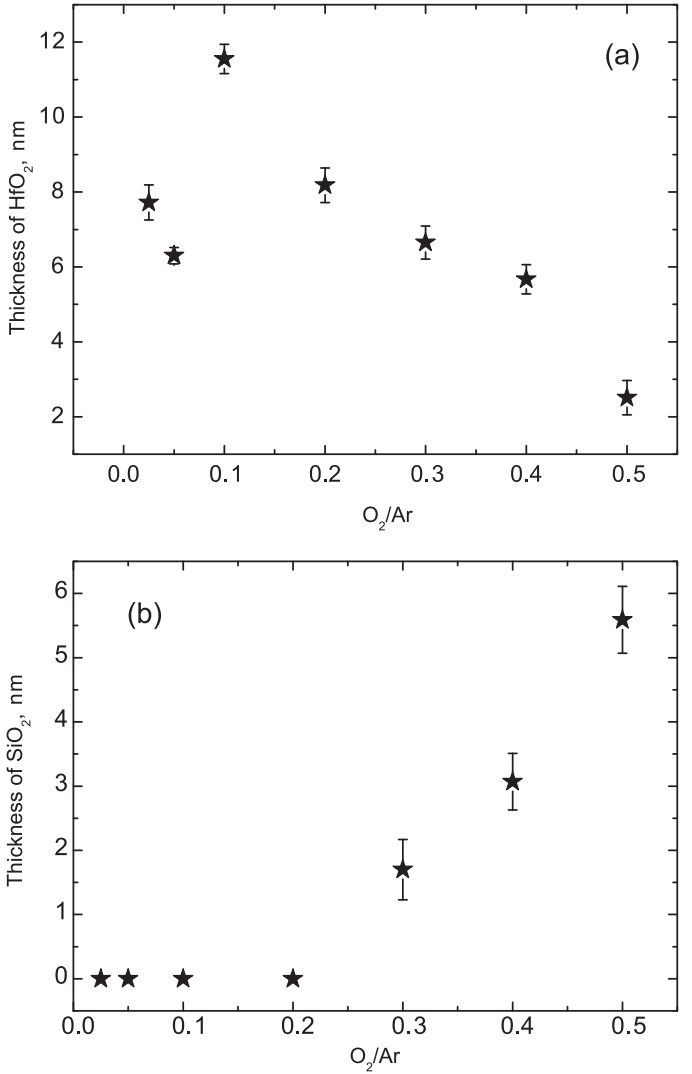

Fig. 7. Change in film thickness with respect to $\mathrm{O}_{2} / \mathrm{Ar}$ gas ratio increment at $P=30 \mathrm{~W}$ for (a) $\mathrm{HfO}_{2}$, (b) $\mathrm{SiO}_{2}$.

sputtered Hf atoms with the effect of gradually increased sputtering powers gain more energy, they probably hit to the substrate with higher velocities resulting in some defect in the film already grown on the substrate. Second possible reason is that Ar ions with elevated energies can remove bigger sized particles from Hf target, therefore, resulting in a porous film formation on the substrate. The observed reduction in refractive index of grown Hf-oxide film as a result of increased sputtering power has also been reported by some other groups [39]. Therefore, the sputtering power should be suitably arranged in order not to give any detectable damage to the surface of already grown film, as well as to be able to realize the growth of dense enough high- $\kappa$ thin film. Dependence of refractive index on applied sputter power is described with an exponentially decaying function having a fit relation of $n=1.445+0.453 e^{- \text {(Sputter power)/28.101 }}$. Since the bulk refractive index value of 2.1 for $\mathrm{HfO}_{2}$ is obtained at the sputter power of $\sim 26.8 \mathrm{~W}$ as a result of the best fit equation, our experimentally predicted value of $30 \mathrm{~W}$ for the best sputtering power choice is, accordingly, in a very well estimation range.

Fig. 7(a) shows the thickness of grown $\mathrm{HfO}_{2}$ layer with respect to $\mathrm{O}_{2} / \mathrm{Ar}$ gas ratio ranging from 0.025 to 0.5 . It is apparent that the thickness of grown film is found to be first increasing till $\mathrm{O}_{2}$ to Ar gas ratio reaches to $\sim 0.1$ and, after then, it decreases again as a result of an increment of the oxygen quantity in the $\mathrm{O}_{2} / \mathrm{Ar}$ gas ratio. Specifically, the thickness of grown film is decreased in an exponential manner when $\mathrm{O}_{2} / \mathrm{Ar}$ gas ratio is getting increased from 0.1 to $\sim 0.2$. Therefore, $\mathrm{HfO}_{2}$ thickness reaches a maximum value at $\sim 0.1$ gas ratio. Fig. 7 (b) shows the thickness of unintentional grown $\mathrm{SiO}_{2}$ interfacial layer with respect to $\mathrm{O}_{2} / \mathrm{Ar}$ gas ratio. Since $\mathrm{HfO}_{2}$ has the poor barrier property to oxygen diffusion [40], it is clear that while the thickness of $\mathrm{HfO}_{2}$ layer get shrinks for increasing amount of oxygen gas, the thickness of $\mathrm{SiO}_{2}$ starts to be significant when 


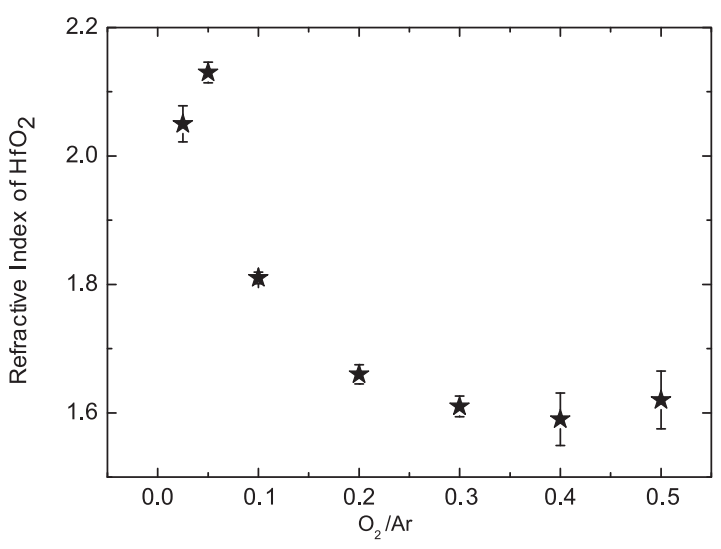

Fig. 8. Change in refractive index of grown hafnium-oxide film as a function of $\mathrm{O}_{2} / \mathrm{Ar}$ gas ratio for $P=30 \mathrm{~W}$ at the wavelength of $632 \mathrm{~nm}$.

$\mathrm{O}_{2} / \mathrm{Ar}$ gas ratio exceeds 0.2. Moreover, there is a linear increase in the $\mathrm{SiO}_{2}$ thickness when the gas ratio is higher than 0.2 which is supported by FTIR analysis. As a result, $\mathrm{O}_{2} / \mathrm{Ar}$ gas ratio needs to be smaller than 0.2 in order to be able to grow only $\mathrm{HfO}_{2}$ but not $\mathrm{SiO}_{2}$.

Fig. 8 shows the refractive index of grown $\mathrm{HfO}_{2}$ layers with respect to $\mathrm{O}_{2}$ to $\mathrm{Ar}$ gas ratio. It is apparent from Fig. 3(a) that the gas ratio affects the state of crystalline structure of grown $\mathrm{HfO}_{2}$ films, i.e., ranging from amorphous for the lowest to polycrystalline to the highest oxygen contents. We observed that optical refractive index decreases while the oxygen gas content. This indicates that the films with low oxygen content gas ratio have amorphous structure as also confirmed by GIXRD measurements. It is well known from the literature that an increase in the level of disorderness has a positive effect on the refractive index of thin films [41]. Therefore, the amorphous structured films obtained with the lower oxygen contents had higher refractive index. It is apparent that the refractive index value being 2.13 for the $\mathrm{O}_{2} / \mathrm{Ar}$ gas ratio of 0.05 is extremely close to that of $\mathrm{HfO}_{2}$ 's bulk value $(n=2.1)$.

Fig. 9 gives the dielectric constant's real part for $\mathrm{HfO}_{2}$ layers with respect to $\mathrm{O}_{2} / \mathrm{Ar}$ gas ratio. Real part of the dielectric constant of hafnium-oxide film depend the amount of the gas ratio on an exponentially decaying function satisfying a best fit equation of $\varepsilon_{1}=2.1577 e^{\left(-\left(\mathrm{O}_{2} / \mathrm{Ar}\right) / 0.2039\right)}+3.1847$. The highest dielectric constant was obtained for the lowest $\mathrm{O}_{2} / \mathrm{Ar}$ gas ratio value of $\sim 0.1$. The film is transparent in the wavelength range of $300-850 \mathrm{~nm}$, therefore, the imaginary part of dielectric constant $\left(\varepsilon_{2}\right)$ goes to zero (no extinction coefficient), i.e., $\varepsilon_{1}$ varies with square of refractive index.

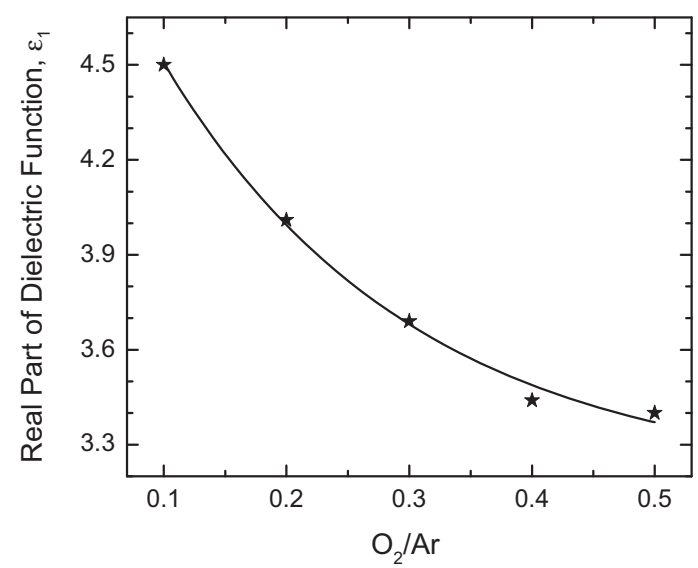

Fig. 9. Real part of dielectric constant of grown hafnium-oxide layer as a function of $\mathrm{O}_{2} / \mathrm{Ar}$ gas ratio at the wavelength of $632 \mathrm{~nm}$.
Results of increasing oxygen amount in $\mathrm{O}_{2} / \mathrm{Ar}$ gas ratio can be summarized as follows: (i) increased amount of oxygen gas in the vacuum chamber is possibly resulting in an increment of the scattering centers for the sputtered Hf atoms and, therefore, preventing these sputtered $\mathrm{Hf}$ atoms from reaching to the substrate, (ii) oxygen diffusion into the interfacial region may lead to the formation of undesired interfacial layer between high-к thin film and Si substrate. Therefore, increased amount of oxygen leads to not only the variations in the hafnium-oxide film thickness but also formation of a porous film structure as well as an uncontrollable interfacial layer. As a result, the oxidation process is found to be more effective at lower oxygen contents of $\mathrm{O}_{2} / \mathrm{Ar}$ ratio between 0.05 and 0.1 .

\section{Conclusion}

We have studied the optical and structural properties of $\mathrm{HfO}_{2}$ high- $\kappa$ thin films produced by magnetron sputtering and discussed how these properties are affected by the $\mathrm{O}_{2} / \mathrm{Ar}$ gas ratio as well as applied sputtering power during deposition. FTIR and SE analysis of the as-deposited samples are used in our work to monitor the interfacial layer formation, while GIXRD measurements are used to determine the crystalline structure of films. Since it is desirable that the high-к dielectric films have amorphous structure without any suboxide film at the interface and that they have the refractive index close to bulk value that of $\mathrm{HfO}_{2}(n=2.1$ at $632 \mathrm{~nm})$, we have drawn the conclusion that the film deposited $30 \mathrm{~W}$ applied power combined with $0.05-0.1$ gas ratio satisfied the expected film quality.

It is concluded that the crystalline structure, thickness and the optical properties of the grown films depend strongly on the deposition parameters. The best rf sputtering oxidation conditions at room temperature in terms of structural and optical parameters of grown $\mathrm{HfO}_{2}$ films is found as $30 \mathrm{~W}$ of sputtering power combined with oxygen to argon gas ratio $\left(\mathrm{O}_{2} / \mathrm{Ar}\right)$ between 0.05 and 0.1 .

\section{Acknowledgments}

This work was financially supported by TUBITAK with the project number of 107T117 and by the Izmir Institute of Technology with BAP project number of 2008IYTE37.

\section{References}

[1] A. Taube, R. Mroczynski, K. Korwin-Mikke, S. Gieraltowska, J. Szmidt, A. Piotrowska, Effect of the post-deposition annealing on electrical characteristics of MIS structures with $\mathrm{HfO}_{2} / \mathrm{SiO}_{2}$ gate dielectric stacks, Mater. Sci. Eng. B 177 (2012) 1281-1285.

[2] G. He, L.D. Zhang, G.W. Meng, G.H. Li, G.T. Fei, X.J. Wang, J.P. Zhang, M. Liu, Q. Fang, I.W. Boyd, Composition dependence of electronic structure and optical properties of $\mathrm{Hf}_{1-x} \mathrm{Si}_{x} \mathrm{O}_{y}$ gate dielectrics, J. Appl. Phys. 104 (2008), 1041161-104116-7.

[3] G. He, L.D. Zhang, G.H. Li, M. Liu, L.Q.Zhu, S.S. Pan, Q. Fang, Spectroscopic ellipsometry characterization of nitrogen-incorporated $\mathrm{HfO} 2$ gate dielectrics grown by radio-frequency reactive sputtering, Appl. Phys. Lett. 86 (2005), 232901$1-232901-3$

[4] G. He, L.Q. Zhu, M. Liu, Q. Fang, L.D. Zhang, Optical and electrical properties of plasma-oxidation derived $\mathrm{HfO}_{2}$ gate dielectric films, Appl. Surf. Sci. 253 (2007) 3413-3418.

[5] M. Toledano-Luque, F.L. Martinez, E. San Andres, A. del Prado, I. Martil, G. Gonzalez-Diaz, W. Bohne, J. Rohrich, E. Strub, Physical properties of high pressure reactively sputtered hafnium oxide, Vacuum 82 (2008) 1391-1394.

[6] J. Aarik, H. Mandar, M. Kirm, L. Pung, Optical characterization of $\mathrm{HfO}_{2}$ thin films grown by atomic layer deposition, Thin Solid Films 466 (2004) 41-47.

[7] T.J. Bright, J.I. Watjen, Z.M. Zhang, C. Muratore, A.A. Voevodin, Optical properties of $\mathrm{HfO}_{2}$ thin films deposited by magnetron sputtering: from the visible to the far-infrared, Thin Solid Films 520 (2012) 6793-6802.

[8] G. He, M. Liu, L.Q. Zhu, M. Chang, Q. Fang, L.D. Zhang, Effect of postdeposition annealing on the thermal stability and structural characteristics of sputtered $\mathrm{HfO}_{2}$ films on $\mathrm{Si}(100)$, Surf. Sci. 576 (2005) 67-75.

[9] Q. Fang, J.-Y. Zhang, Z. Wang, M. Modreanu, B.J. O’Sullivan, P.K. Hurley, T.L. Leedham, D. Hywel, M.A. Audier, C. Jimenez, J.-P. Senateur, I.W. Boyd, Interface 
of ultrathin $\mathrm{HfO}_{2}$ films deposited by UV-photo-CVD, Thin Solid Films 453-454 (2004) 203-207.

[10] R. Thielsch, A. Gatto, J. Heber, N. Kraiser, A comparative study of the UV optical and structural properties of $\mathrm{SiO}_{2}, \mathrm{Al}_{2} \mathrm{O}_{3}$, and $\mathrm{HfO}_{2}$ single layers deposited by reactive evaporation, ion-assisted deposition and plasma ion-assisted deposition, Thin Solid Films 410 (2002) 86-93.

[11] A. Deshpande, R. Inman, G. Jursich, C. Takoudis, Characterization of hafnium oxide grown on silicon by atomic layer deposition: interface structure, Microelectron. Eng. 83 (2006) 547-552.

[12] J. Kim, S. Kim, H. Kang, J. Choi, H. Jeon, M. Cho, K. Chung, S. Back, K. Yoo, C. Bae, Composition, structure, and electrical characteristics of $\mathrm{HfO}_{2}$ gate dielectrics grown using the remote- and direct-plasma atomic layer deposition methods, J. Appl. Phys. 98 (2005) 094504.

[13] J. Zhu, Z.G. Liu, Y. Feng. Thermal stability and electrical properties of pulsed laser-deposited Hf-silicate thin films for high- $k$ gate dielectric applications, J. Phys. D: Appl. Phys. 36 (2003) 3051-3056.

[14] G. Aygun, R. Turan, Electrical and dielectrical properties of tantalum oxide films grown by Nd:YAG laser assisted oxidation, Thin Solid Films 517 (2008) 994-998.

[15] G. Aygun, E. Atanassova, A. Alacakir, L. Ozyuzer, R. Turan, Oxidation of Si surface by a pulsed Nd:YAG laser. J. Phys. D: Appl. Phys. 37 (2004) 1569-1575.

[16] K. Yamamoto, S. Hayashi, M. Niwa, M. Asai, S. Horii, H. Miya, Electrical and physical properties of $\mathrm{HfO}_{2}$ films prepared by remote plasma oxidation of $\mathrm{Hf}$ metal, Appl. Phys. Lett. 83 (2003) 2229-2231.

[17] R. Tan, Y. Azuma, I. Kojima, Suppression of interfacial diffusion by a predeposited Hf metal layer on $\mathrm{SiO}_{2} / \mathrm{Si}$, Appl. Surf. Sci. 222 (2004) 346-350.

[18] O. Tuna, Y. Selamet, G. Aygun, L. Ozyuzer, High quality ITO thin films grown by DC and RF sputtering without oxygen, J. Phys. D: Appl. Phys. 43 (2010) 055402.

[19] G. Aygun, A. Cantas, Y. Simsek, R. Turan, Effects of physical growth conditions on the structural and optical properties of sputtered grown thin $\mathrm{HfO}_{2}$ films, Thin Solid Films 519 (2011) 5820-5825.

[20] G. Tian, S. Wu, K. Shu, L. Qin, J. Shao, Influence of deposition conditions on the microstructure of oxides thin films, Appl. Surf. Sci. 253 (2007) 8782-8787.

[21] Z. Xie, Y. Lai, Effects of oxygen partial pressure on the sputtered hafnium oxide thin films for resistive random-access memory, Adv. Mater. Res. 529 (2012) 49-52.

[22] G. Aygun, I. Yildiz, Interfacial and structural properties of sputtered $\mathrm{HfO}_{2}$ layers, J. Appl. Phys. 06 (2009) 014312.

[23] ICDD database: D. Grier, G. McCarthy, North Dakota State University, Fargo, North Dakota, USA, ICDD, Grant in Aid (1991).

[24] G. He, M. Liu, L.Q. Zhu, M. Chang, Q. Fang, L.D. Zhang, Spectroscopic ellipsometry characterization of nitrogen-incorporated $\mathrm{HfO}_{2}$ gate dielectrics grown by radio-frequency reactive sputtering, Surf. Sci. 576 (2005) 67-69.

[25] K. Kukli, M. Ritala, J. Sundqvist, J. Aarik, J. Lu, T. Sajavaara, M. Leskela, A. Harsta, Properties of hafnium oxide films grown by atomic layer deposition from hafnium tetraiodide and oxygen, J. Appl. Phys. 92 (2002) 5698-5703.

[26] Atanassova, G. Aygun, R. Turan, Tz Babeva, Structural and optical characteristics of tantalum oxide grown by pulsed Nd:YAG laser oxidation, J. Vac. Sci. Technol. A 24 (2006) 206-211.
[27] L.-P. Feng, Z.-T. Liu, Y.-M. Shen, Compositional, structural and electronic characteristics of $\mathrm{HfO}_{2}$ and $\mathrm{HfSiO}$ dielectrics prepared by radio frequency magnetron sputtering, Vacuum 83 (2009) 902-905

[28] G. Aygun, G. Roeder, T. Erlbacher, M. Wolf, M. Schellenberger, L. Pfitzner, Impact of temperature increments on tunneling barrier height and effective electron mass for plasma nitrided thin $\mathrm{SiO}_{2}$ layer on a large wafer area, J. Appl. Phys. 108 (2010) 073304

[29] L. Pereira, A. Marques, H. Augas, N. Nedev, S. Georgiev, E. Fortunato, R. Martins, Performances of hafnium oxide produced by radio frequency sputtering for gate dielectric application, Mater. Sci. Eng. B 109 (2004) 8993.

[30] D.A. Neumayer,, E. Cartier, Materials characterization of $\mathrm{ZrO}_{2}-\mathrm{SiO}_{2}$ and $\mathrm{HfO}_{2}-\mathrm{SiO}_{2}$ binary oxides deposited by chemical solution deposition, J. Appl. Phys. 90 (2001) 1801-1808.

[31] M. Toledano-Luque, E. San Andres, A. del Prado, I. Martil, M.L. Lucia, G. GonzalezDiaz, High-pressure reactively sputtered $\mathrm{HfO}_{2}$ : composition, morphology, and optical properties, J. Appl. Phys. 102 (2007) 044106-44111.

[32] G. He, L.D. Zhang, Q. Fang, Silicate layer formation at $\mathrm{HfO}_{2} / \mathrm{SiO}_{2} / \mathrm{Si}$ interface determined by X-ray photoelectron spectroscopy and infrared spectroscopy, J. Appl. Phys. 100 (2006), 083517-1-083517-5.

[33] G.B. Alers, D.J. Werder, Y. Chabal, H.C. Lu, E.P. Gusev, E. Garfunkel, T. Gustafsson, R.S. Urdahl, Intermixing at the tantalum oxide/silicon interface in gate dielectric structures, Appl. Phys. Lett. 73 (1998) 1517-1519.

[34] V. Singh, S.K. Sharma, D. Kumar, R.K. Nahar, Study of rapid thermal annealing on ultra thin high-k $\mathrm{HfO}_{2}$ films properties for nano scaled MOSFET technology, Microelectron. Eng. 91 (2012) 137-143.

[35] T.C. Chen, C.Y. Peng, C.H. Tseng, M.H. Liao, M.H. Chen, C.I. Wu, M.Y. Chern, P.J. Tzeng, C.W. Liu, Characterization of the ultra-thin $\mathrm{HfO}_{2}$ and Hf-silicate grown by atomic layer deposition, IEEE Trans. Electron Devices 54 (2007) 759776.

[36] J.C. Hackley, T. Gougousi, Properties of atomic layer deposited $\mathrm{HfO}_{2}$ thin films Thin Solid Films 517 (2009) 6576-6583.

[37] H.G. Tompkins, W.A. McGahan, Spectroscopic Ellipsometry and Reflectometry, Wiley, New York, 1999.

[38] H. Fujiwara, Spectroscopic Ellipsometry Principles and Applications, John Wiley \& Sons, Chichester, UK, 2007.

[39] S. Duenas, H. Castan, H. Garcia, A. Gomez, L. Bailon, M. Toledano-Luque, I. Martil, G. Gonzales-Diaz, Electrical properties of high-pressure reactive sputtered thin hafnium oxide high- $k$ gate dielectrics, Semicond. Sci. Technol. 22 (2007) $1344-1351$.

[40] G. He, L.D. Zhang, G.W. Meng, G.H. Li, Q. Fang, J.P. Zhang, Temperaturedependent structural stability and optical properties of ultrathin Hf-Al-O films grown by facing-target reactive sputtering, J. Appl. Phys. 102 (2007), 094103 1-094103-5.

[41] G. Aygun, E. Atanassova, R. Turan, Tz Babeva, Reflectance spectra and refractive index of a Nd:YAG lazer-oxidized Si surface, Mater. Chem. Phys. 89 (2005) 316-320. 